\title{
Interactive comment on "A process-based diagnosis of catchment coevolution in volcanic landscapes: synthesis of Newtonian and Darwinian approaches" by Takeo Yoshida and Peter A. Troch
}

Takeo Yoshida and Peter A. Troch

takeoys@affrc.go.jp

Received and published: 3 August 2016

[Comment] This study looks at the relations between topography and streamflow characteristics. It does so by using the interesting approach to switch catchments parameterizations and input time series. As much as I like the approach there are a number of concerns with the current version of the study:

[Response] Thank you very much for taking the time to review our manuscript and for providing insightful comments. All of the concerns you raised have been addressed below. First, it is important to note that this study look at the relations between streamflow 
characteristics and the age of the catchment, not with topography. We set out to infer the physical processes governing the catchment evolution (as it ages) with the aid of a process-based hydrological model. The model parameters are identified to reproduce the behavior of sub-models properly, rather than generating better objective functions (high NSE for instance). We examined several hydrological signatures including the annual and seasonal water partitioning at the land surface (Fig.3) and the slope of the flow duration curves (Fig.4). The parameter uncertainty is thus out of the scope of this paper.

[Comment] There is a fundamental assumption that the input time series do not influence the parameter values. While one might hope for this (the parameters should represent the physical characteristics after all), many studies have shown, that parameter values actually are related to variables such as mean annual precip. This issue needs to be addressed as it could largely influence the conclusions from this study.

[Response] We agree with you that the input time series will affect the parameter values, but since we used all available data (20 years) there is no other time period that we could use to see how sensitive the parameters are to the time series selected. Moreover, we don't know how this will affect the results, since we swap climates and models, and we look at how robust the predictions of certain signatures are to internal and external characteristics.

[Comment] P3L86: it is unclear why 8 out of 14 catchments were selected; this may sound a bit like cherry-picking. Please explain why/how only 8 catchments were selected here.

[Response] We selected eight catchments so that the age and climate gradients are maintained as the original samples. First, we plotted the age and aridity index of the study catchments of our previous study (Yoshida and Troch, 2016; Fig.1 in this response manuscript) and selected 4 clusters of catchments, i.e., the youngest $(\sim 0.2$ $\mathrm{Ma})$, younger $(\sim 3 \mathrm{Ma})$, older $(\sim 10 \mathrm{Ma})$ and the oldest $(\sim 80 \mathrm{Ma})$. We then selected

Printer-friendly version

Discussion paper 
three catchments each from the 'younger' and 'older' clusters, each of which have different climate properties, and one catchment each from the 'youngest' and 'oldest'. Previous study of Carrillo et al. (2011) suggested the poor representation of hsB-SM in snow-dominant catchments; we thus selected both ASE and SIM catchments that are almost identical in terms of the age and aridity index, but are different as one of them is snow-dominant (ASE) while other is not (SIM). We will add text to the revised manuscript to make our selection procedure more transparent.

[Comment] P5L138: The consideration of parameter uncertainty is not convincing. The sequential calibration is highly sensitive to the order of the parameter in calibration, and this order is not clearly described/motivated. Of course, this approach apparently reduces parameter interactions/uncertainty, but it does so by only investigating part of the parameter space. Just because one is not looking everywhere, does not mean parameter uncertainty is really reduced! I would recommend to consider parameter uncertainty explicitly by allowing for different parameter sets using some type of Monte Carlo approach (resulting in ranges of simulated streamflow characteristics).

[Response] First, we used the same approach as in Carrillo et al (2011). The reason for this way of estimating the parameter values is that we want to make sure that each subcomponent of the model (shallow subsurface flow, deep groundwater, root zone dynamics etc) is behaving properly, we are not after a globally optimal model that may have the objective function right (high NSE for instance) but does a lousy job in getting the response of the subcomponents right. Monte Carlo simulations are thus out of the scope of this paper.

[Comment] Much of the analysis is based on the assumption that the model realistically can reproduce the observed runoff. There are two issues with this. The performance of the model in terms of NSE is not fully demonstrated, it is only mentioned that the NSE values were smaller 0.5 in many catchments (P9L232). This sounds rather alarming to me! Furthermore, even higher NSE values would not ensure that the different streamflow characteristics (signatures) would be realistically reproduced. It also remains un-

Printer-friendly version

Discussion paper
Interactive

comment 
clear how the different goodness-of-fit measures were combined (weighted mean). As the results largely depend on the parameterization and model performance, all these above issues are crucial for this study and need to be better addressed/described.

[Response] The signature under investigation is the slope of the FDC, because this is the hydrologic signature that evolves with the age of the catchments. Figure 4 illustrates that the models do a good job in simulating this signature.

[Comment] I am a bit confused by the term coevolution. This sounds fancy, but does the manuscript really deal with coevolution? I don't think so. Even if there is a relationship between topography and flow characteristics this does show necessarily any coevolution. Please clarify this term in the context of the manuscript (or omit it). The second part of the title (Newtonian/Darwinian) remains a total mystery to me, please explain what is meant here.

[Response] Again, the paper is about the relation between the age of the catchments and flow characteristics, not about topography. The paper is about coevolution because we ask the question why does the slope of the flow duration curve changes predictably with age, and can we discover what catchment characteristics are responsible for this evolution? We build on the work of Jefferson et al. (2010) and Yoshida and Troch (2016) where this relationship was observed empirically, here we want to understand the mechanisms behind this, by testing the hypothesis put forward previously that with age the soils develop a shallow semi-impermeable layer that leads to faster flow paths and steeper slopes of the FDC. The Newtonian part refers to the mechanistic physics-based model that we use, the Darwinian part refers to the inter-comparison of catchments and the postulation of a hypothesis (and sequentially testing of this hypothesis) about what explains the difference in response and how it is related to catchment age (see Harman and Troch, 2014).

[Reference] Carrillo, G., Troch, P. A., Sivapalan, M., Wagener, T., Harman, C., and Sawicz, K.: Catchment classification: hydrological analysis of catch- 
ment behavior through process-based modeling along a climate gradient, Hydrology and Earth System Sciences, 15, 3411-3430, doi:10.5194/hess-15-3411-2011, http://dx.doi.org/10.5194/hess-15-3411-2011, 2011. Harman, C. and Troch, P. A.: What makes Darwinian hydrology" Darwinian"? Asking a different kind of question about landscapes, Hydrology and Earth System Sciences, 18, 417-433, 2014. Jefferson, A., Grant, G., Lewis, S., and Lancaster, S.: Coevolution of hydrology and topography on a basalt landscape in the Oregon Cascade Range, USA, Earth Surface Processes and Landforms, 35, 803-816, 2010.

Interactive comment on Hydrol. Earth Syst. Sci. Discuss., doi:10.5194/hess-2016-263, 2016. 


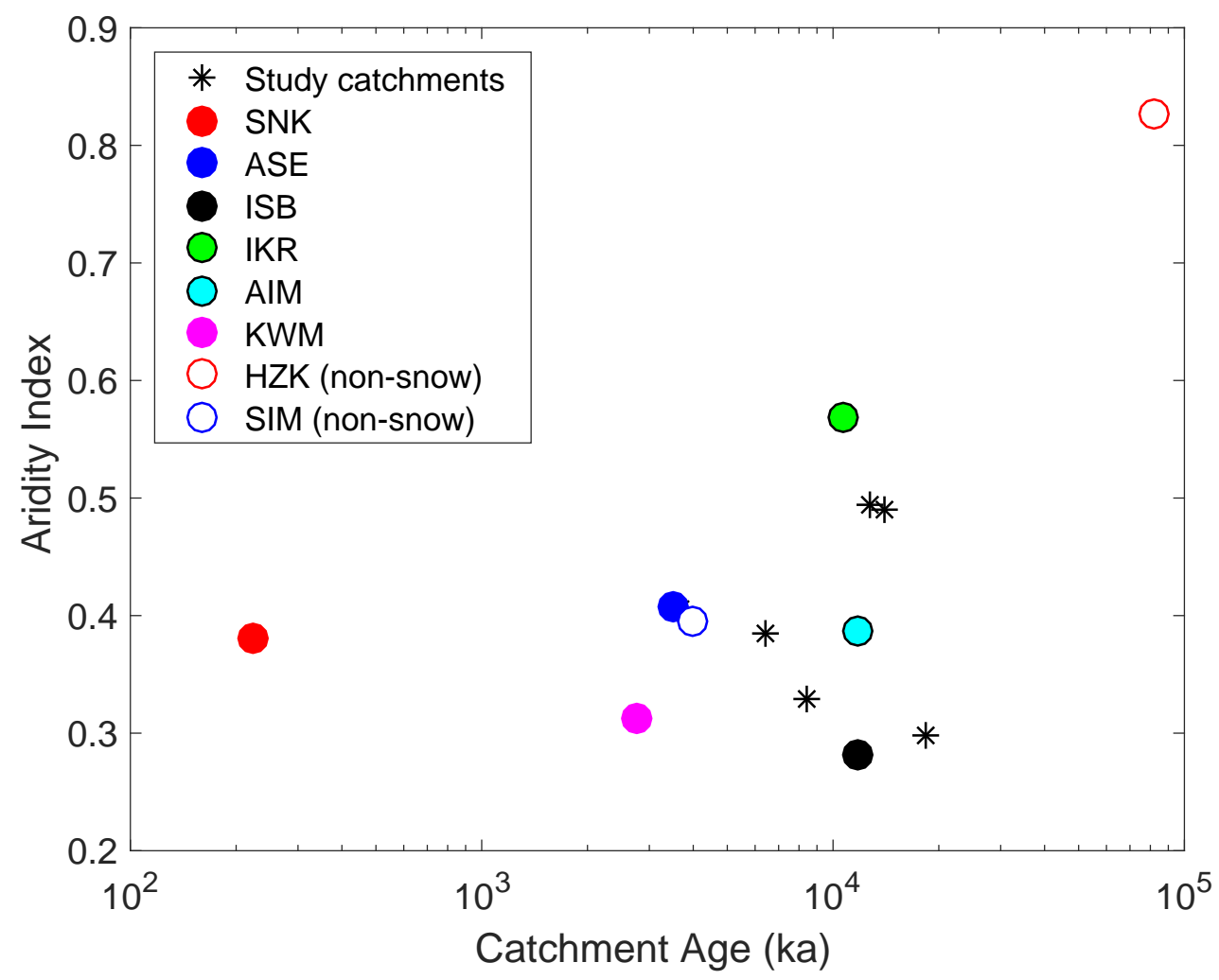

Interactive

comment

Fig. 1. Selected eight catchments (circles) from the study catchments (stars indicate nonselected catchments) of Yoshida and Troch (2016) 